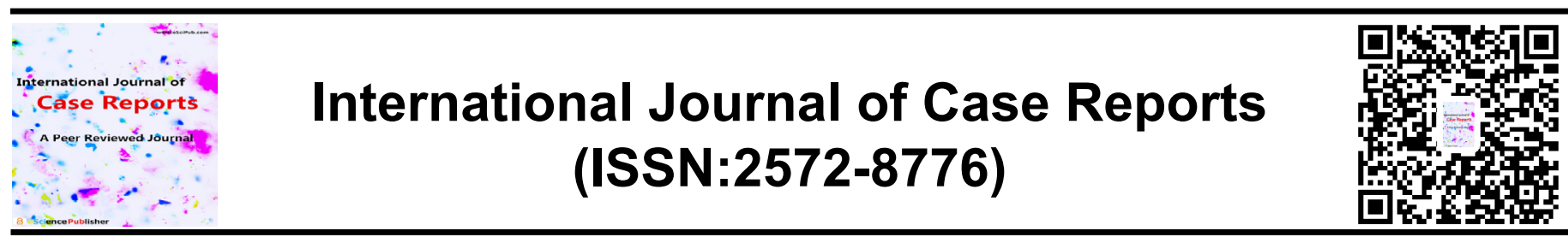

\title{
Case of severe head trauma caused by a car accident
}

\section{Liliana Dragomir $^{1 *}$; Virginia MARINA ${ }^{2}$; Mihaela Anghele ${ }^{3}$}

${ }^{1}$ MD. Liliana DRAGOMIR, CLINICAL-MEDICAL Department, « Dunarea de jos » University of Galati, Faculty of Medicine and Pharmacy, Galati, Romania.

${ }^{2}$ PH.D. Virginia MARINA, MEDICAL Department of Occupational Health, « Dunarea de jos » University of Galati, Faculty of Medicine and Pharmacy, Galati, Romania.

${ }^{3}$ MD. Mihaela ANGHELE, CLINICAL-MEDICAL Department, « Dunarea de jos » University of Galati, Faculty of Medicine and Pharmacy, Galati, Romania.

\section{ABSTRACT}

According to the literature, the term "poly-trauma" was first *Correspondence to Author: used by Tscherne et.al, in 1966 for patients who demonstrated a combination of at least two "severe head, chest or abdominal injuries" or "one of them in association with an extremity injury. Trauma, despite all efforts to control its impact, is now a recurrent cause of high incidence of mortality and morbidity worldwide. Studies show that road traffic injuries are currently the leading cause of death among people aged between 1 and 45-years old. Only 21 countries in the WHO (World Health Organization), European Region have implemented legislative changes regulating drink-driving, with blood alcohol test limits varying between $\leq 0.02 \mathrm{~g} / \mathrm{dl}$ and $\leq 0.05 \mathrm{~g} / \mathrm{dl}$.

\section{INTRODUCTION:}

It is well known that poly-trauma is a challenge for the clinician, regardless of specialization. In this regard, we wanted to present this case to raise an alarm on the approach to poly-trauma, which involves, in addition to the pre-hospital, hospital and follow-up stages, the follow-up of victims after discharge, in an attempt to identify possible causes of death and their prevention.

\section{CASE PRESENTATION:}

In this clinical case, we present the outcome of a 44-year-old patient who presented to the emergency room with multiple trauma injuries resulting from a road traffic accident. During her admission to the neurosurgery department of the Emergency "Sfântul Apostol Andrei", Clinical Hospital of Galati, the patient 
underwent numerous clinical and imaging investigations, which provided findings statistically associated with increased mortality and morbidity rates.

\section{CONCLUSION:}

The management of patients presenting with poly-trauma is continuous, dynamic and extremely challenging in terms of the staff involved, the multidisciplinary team that will monitor the patient's progress. All this is a goal that can only be achieved through good interdisciplinary and interhospital communication, providing standards of care and quality-oriented cooperation, increasing efficiency through the use of existing resources, regulating the financial aspects of inter-hospital transfers, cooperating in a common system of continuous training.

Keywords: Poly-trauma, traumatic brain injury, multiple fracture sites, Bronchopneumonia, Pulmonary contusions

\section{Background}

In 1975, Border defined the poly-trauma patient as "any patient with two or more significant injuries" [1]. Poly-trauma has a varied and inhomogeneous content, which is why the terminology "poly-trauma" can only be used as a final diagnosis after objective quantification of the degree of severity of the injuries ${ }^{[2]}$.

Oestern characterized the notion of "polytrauma" following patients with the following characteristics: two or more injuries, one of which is potentially life-threatening, isolated but potentially life-threatening injuries, which they referred to as "barotrauma" [3].

Until recently, the notion of poly-trauma has been defined by a high Injury Severity Score (ISS), which has also been used to define terms such as 'multiple trauma' and 'major trauma'. Conventionally, internationally the mortality prevalence is considered to reach values above $10 \%$ when the ISS threshold is greater than or equal to 16 , but there are studies that record variations in ISS score values (greater than 15,
$17,18$ or even 25$)$ thus defining poly-trauma in different ways ${ }^{[4,5,6]}$.

In analyses carried out by Euro-stat, in 2016 at the European level there were approximately 50 deaths per 1 million inhabitants due to road accidents, in contrast to global values of 174 deaths per 1 million inhabitants [7]. Also, in 2016 the number of road deaths decreased by $2 \%$, with 25,500 deaths occurring this year, 600 fewer than in 2015 and 6,000 fewer than in 2010. A downward trend can be seen when it comes to the incidence of accidents, with the number of fatalities falling considerably between 2001 and 2010 , by around $43 \%$.

Statistics provided by the United States give a bleak picture of the incidence of TBI, as it is the leading cause of death reported in patients younger than 45 years ${ }^{[8,9]}$. Data from the last 20 years demonstrate the effectiveness of regionalization of trauma care in optimizing the treatment of poly-trauma patients, and also show the effectiveness of this approach in reducing mortality ${ }^{[10]}$. 
To provide this level of care, numerous resources are required, both for maintaining a 24-hour emergency service readiness, and also continuous availability of specific ambulance cars and helicopters. Trauma centers also require specific structural features, such as the availability of intensive care units, trauma units, imaging equipment such as $\mathrm{CT}$, and access to a blood bank. In addition to special equipment, they also require the availability of specifically trained staff, such as doctors specializing in: emergency medicine, vascular surgery, anesthetists, radiologists, neurosurgeons, nurses, orthopedists. ${ }^{[11]}$

The care of poly-trauma patients is still a major and highly complex problem in the public institutions card, both locally and worldwide. In order to implement a specific and effective treatment in the treatment of poly-trauma, it is necessary to integrate all the specific variables of each region, such as: cultural, social and historical context. This difference is mainly produced by the presence of trauma centers at national level, which explains the lower mortality rates in the USA and some European countries $[12,13]$.

\section{Case presentations}

On 22.10.2019, the SMURD (mobile emergency resuscitation and release service) crew from Galati County took over a female patient, victim of a road accident. At $02.33 \mathrm{am}$, a female 44year-old patient was brought by an SMURD crew to the Emergency Reception Unit of the "Sfântul Apostol Andrei" Hospital of Galati. Preliminary data obtained from the investigators revealed that the patient was part of a road accident in Galati County, most likely due to failure to adapt speed to road and weather conditions. As a result, the car in which the victim was travelling left the carriageway and collided directly with a tree.

At the time of the emergency unit's assessment, the patient had the following neurological and cardiopulmonary parameters: GCS (Glasgow Criterion Scale) score of 3 points, respiratory rate -16 breaths $/ \mathrm{min}, \mathrm{SpO} 2$ of $100 \%$ in atmospheric air, AV of 85 Heart rate/minute, Blood Pressure equal to $120 / 60 \mathrm{mmHg}$. Clinical and imaging examinations performed at the time of admission revealed that the patient had numerous facial bruises, a temporal-parietal contusion, and right upper limb involvement. A thorough evaluation revealed left temporalparietal subarachnoid hemorrhage, right temporal-parietal, the adjacent part of axillary and the adjacent part of orbital epi-cranial hematoma on the same side, and the victim had a skull base fracture. The blood collected from the victim during admission to Galati County Hospital contained ethanol at a concentration of $2.7 \mathrm{~g} / \mathrm{L}$.

First intention (22.10.2019 - emergency service) radiological investigations were performed as follows:

- Spine CT scan: no recent post-traumatic bone lesions at the time of examination.

- Native cranium-cerebral CT which detected: left temporal-parietal subarachnoid hemorrhage and more discrete to the right side, preserved median axis, with symmetrical ventricular sinus, reduced dimensions, bilateral cortical relief obliteration. In addition, the patient presented right temporal, parietal, cranial hematoma, surrounded maxillary and surrounded -orbital on the same side. Multiple fracture tracts in the anterior floor of the skull base (accentuated on the right side) with the presence of sinus hemorrhage in the maxillary sinus and ethmoidnasal regions, as well as left mandible fracture (Figure 1).

Thorax CT, abdomen and pelvis with intravenous medium contrast

- Bilateral lung contusions (right-sided emphasis) predominantly basal, minimal left apical-anterior- pneumothorax, right pleural fluid reaction, bilateral rib fractures (Figure 2). At the medial segment of the right middle lobe, a small cavity image is seen, surrounded by an area of possible fibrosis. Liver with increased size, sharp outline, homogeneous structure, no recent posttraumatic lesions, portal vein in hilum about 12 
$\mathrm{mm}$, patent. Spleen, gall bladder, pancreas, adrenal glands, left kidney and right kidney no recent posttraumatic lesions. Renal secretion and excretion present bilaterally, minimal hypotonic pyloric and urethral on right. Urinary

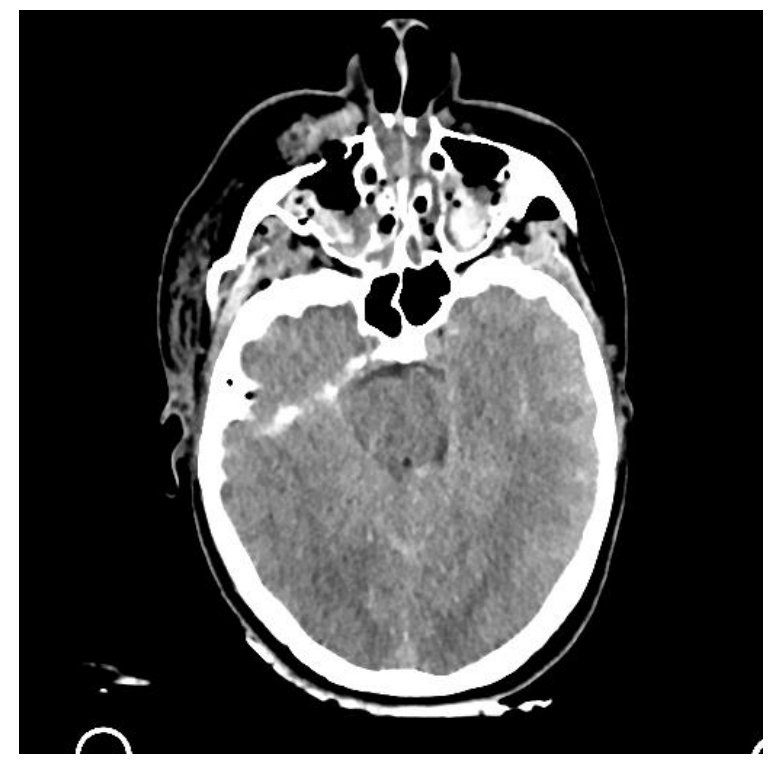

Figure 1: Cranium-cerebral CT of patient

Imaging investigations in conjunction with neurosurgical examination, confirm the cranial CT with a patient in a comatose state, CGS score 3 points, which is why the decision is taken to admit her to the neurosurgery department. In addition, the following was performed:

- Thoracic surgery examination (from thorax CT) - bilateral rib fractures with minimal bilateral pulmonary contusion and right pleural reaction of about $1.2 \mathrm{~cm}$

- Orthopaedic examination: between fingers: wound finger 2-3 left hand with multiple abrasions located on the left hand and forearm for which sutures and subsequent dressing fixation were performed.

- General surgical examination - no visceral organic lesions or presence of fluid inside of peritoneal space.

- ENT (otorhinolaryngology) examination tracheal gold intubate patient, unconscious, normal otoscope examination bilaterally, with removal of left intranasal glass fragments and resolution of bilateral anterior epistaxis. bladder in semi-replete, with catheter. Uterus much enlarged in volume, fibromatous tissue transformed, largest nodule approximately 63 $\mathrm{mm}$ in diameter. No free intra-peritoneal fluid on examination.

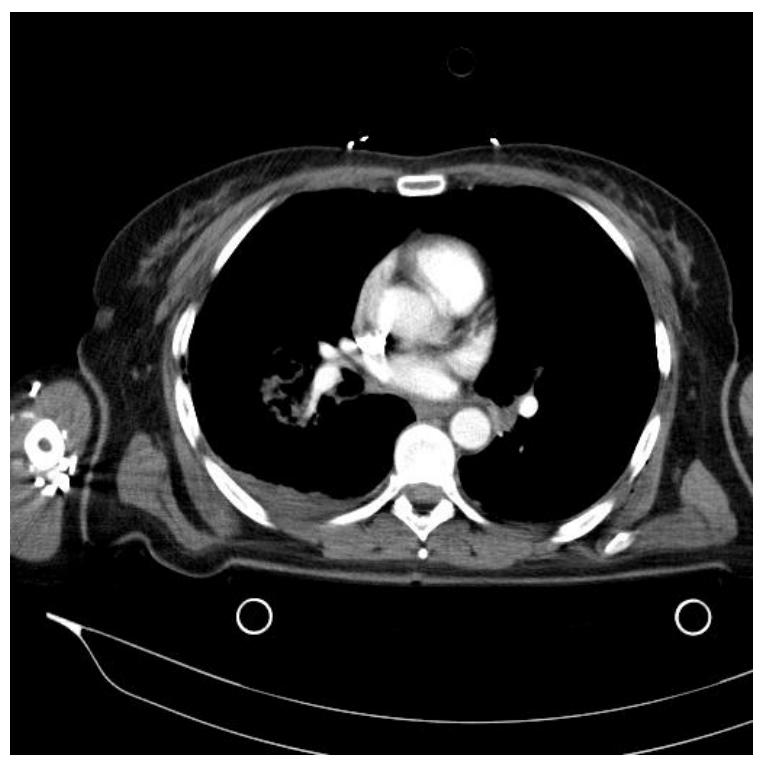

Figure 2: Thorax CT of patient, abdomen and pelvis

The patient was admitted between 22.10.201927.11.2019 in the neurosurgery ward and then transferred to the thoracic surgery ward with the following diagnoses such as:

- Road traffic accident

- Bilateral rib fractures

- Hemorrhagic cerebral contusions

- Right and left post traumatic pleurisy

- In remission: acute respiratory failure; bilateral pulmonary contusions

The patient was admitted to the Neurosurgery Department and underwent repeated imaging and clinical investigations in order to ensure the most correct and prompt management of her pathology.

\section{Discussions}

As can be seen, in the period between 23.10.2019-04.11.2019 the patient underwent multiple imaging investigations as follows:

- On 23.10. 2019 Brain and chest CT with contrast substances were performed again 
- Subsequently a number of 4 control chest Xrays were performed, each time detecting radiological normal heart, but also numerous rib fracture foci and changes in the lung fields. Thus, it can be concluded that since 26.10.2019 (at which time radiological only a bilateral, inferior to the hilum, accentuated pulmonary gap tissue was described, with discreet accentuation on the right side), the patient developed signs of right basal thick tissue of lung and pulmonary emphysema (radiological detected on 01.11.2019 - as a bulky concentric area). Subsequently, on 03.11. 2019, a slightly inhomogeneous right basal opacities were detected, with consecutive right ribsdiaphragmatic sinus voiding.

It is important to mention that on 31.10 .2019 , clinical examinations were performed, which consisted of trachea-bronchial aspirate analysis with culture. It was detected the presence of gram-negative glucose-negative bacilli.

On 05.11.2019 it was decided to perform a surgical intervention for the practice of tracheotomy of necessity. Clinical examination performed at this time indicates the following: serious general condition, with GCS score of 3 points, by eye pupils' examination is noted bilateral myosin, the patient is still with tracheal gold intubate and mechanical ventilation. The patient presents mobile abdomen with respiration, bowel transit in physiological conditions with fixed urinary catheter fitting.

Between 05.11.2019 - 11.11. 2019 continuous monitoring of the patient continued as follows:

Radiological investigations: Thorax $\mathrm{X}$-rays $(n=2)$, abdominal ultrasound $(n=1)$, brain CT $(\mathrm{n}=1)$,

Thorax CT $(n=1)$

- Clinical: blood culture $(n=1)$ and trachea-bronc hial aspirate $(n=1)$

Patient's evolution post tracheotomy, in the neurosurgery department:

06.11.2019:
- Blood culture: coagulase-negative staphylo- coccus

- Brain CT:

$>$ inside the meninx: Small and bilateral hyper densities at this level of the convexity

$>$ fracture of the right maxilla and right sphenoid wing

> bilateral maxillary sinus walls fractures (inside the sinus: fluid collections)

- Thorax CT :

$>$ alveolar condensation process

$>$ bilateral pleurisy

$>$ fracture sites: sternal area and right scapula, multiple rib fractures, bilaterally 07.11 .2019

- bronchial trachea aspirate: Pseudomonas staphylococcus positive 08.11.2019 Thorax X-ray :

$>$ micro-opacities disseminated superiorly bilate- rally

$>$ the right apical heterogeneous

$>$ upper left opacity with a diameter of 15 $\mathrm{mm}$

> rib fractures: right lateral 2;3;4 arches

\subsubsection{0}

Thorax X-ray: lung gap increased with the failure heterogeneous and congestive area surround-ing the heart

Abdominal ultrasound:

$>$ liver with steatosis

$>$ minimal bilateral pleurisy

Subsequently, the patient remained hospitalized in the neurosurgery ward until 27.11.2019, and a seriate control brain CT scan was performed, with no neurosurgical lesions detected.

Then, due to the favorable neurological evolution it was decided to transfer her, to the thoracic surgery ward for specialist treatment, neurological re-evaluation to be performed in 30 days, or as needed. Neurologically, the patient 
received consent to undergo anticoagulation treatment forpulmonary embolism prophylaxis and subsequent mobilization.

On 02.12.2019 the patient was re-evaluated by the ENT doctor who decided that she would require maintenance of the tracheal cannula until the trachea-bronchial secretions disappeared, returning for de-cannulation after 03.12.2019.

Finally, it is decided to discharge the patient on 03.12.2019 with the following recommendations such as:

- Treatment according to prescription

- Return for control after 14 days' post discharge, or if needed

- Return to ENT department for de-cannulation after 03.01.2020

- Avoidance of physical exertion and local trauma

The patient will subsequently die at home on 28.12.2019. Corroboration of the information obtained from the observation sheet, followed up during admission (and all medical records provided and analyzed) with the necropsy findings will conclude that the patient's death was due to bronchopneumonia. This was a complication arising from the evolution of polytrauma with hemorrhaged and meninx-cerebral contusions, viscera-cranium fractures, rib fractures, etc.

\section{Conclusions}

International research in recent decades has hypothesized that motor vehicle accidents are responsible for $75 \%$ of post-traumatic deaths, and that these deaths are most frequently caused by traumatic brain injury (DiScala, Sege, $\mathrm{Li}$ and Reece, 2000), due to extra-cranial complications: gastrointestinal disorders, cardiovascular disorders, coagulopathies, hydro-electrolytic and acid-base imbalances, respiratory disturbances and toxicities).

Clinical studies have shown that bronchopneumonia is the most common respiratory complication of severe traumatic brain injury, with an incidence approaching 2/3 of all cases. ${ }^{[14]}$ The toxic-septic state of patients often begins within the first 8 days of admission, and is often closely related to septic complication phenomena existing at the intracranial level.

For patients in the age group 41-60 years, the highest incidence of abscessed bronchopneumonia is noted, with symptoms that may begin as early as the first day of admission. ${ }^{144}$.

Multidisciplinary approach to poly-trauma is mandatory for the healing evolution of the victims.

Traumatic injuries can associate and aggravate each other, opening vicious circles leading to death.

The case presented was, no exception, to the data that we have in the specialized literature: the patient had an evolution towards exit due to bronchopneumonia.

The particularity of the case was that the patient developed bronchopneumonia after discharge.

\section{Sources of funding}

\section{No funding for the research \\ Ethical approval}

This is not a research study. No ethical approval was necessary.

\section{Consent}

Consent for publication was obtained from patient before discharged.

\section{Author contribution}

DRAGOMIR Liliana - collected the data, wrote the clinical part

MARINA Virginia - wrote the article, processed the pictures; corresponding author

Mihaela ANGHELE -the doctor who received the patient in the emergency room; collaborating physician

Registration of research studies

This is a case report. Is not a research study.

\section{Guarantor}

DRAGOMIR Liliana

ANGHELE Mihaela

Provenance and peer review 
Not commissioned externally peer- reviewed.

\section{Declaration of Competing Interest}

The author decelerates that there is not conflict of interest regarding the publication of this article.

\section{References}

[1] Border, J., LaDuca, J. and Seibel, R., 1975. Priorities in the management of the patient with polytrauma. Prog. Surg., 14, pp.84-120.

[2] Kroupa, J., 1990. K definici polytraumatu a polytraumatismu [Definition of "polytrauma" and "polytraumatism"]. Acta Chir Orthop Traumatol Cech, 57(4), pp.347-360.

[3] Oestern, H., Regel, G., Tscherne, H. and Regel, G., 1997. " Klinische Behandlung Des Schwerverletzten" Unfallchirurgie: Trauma Management. Chapter 9, Berlin, Heidelber, New York : Springer, pp.225-238.

[4] Boyd, C., Tolson, M. and Copes, W., 1987. Evaluating trauma care: The TRISS method. Trauma Score and the Injury Severity Score. J. Trauma, 27, pp.370-378.

[5] Sikand, M., Williams, K., White, C. and Moran, C., 2005. The financial cost of treating polytrauma: Implications for tertiary referral centres in the United Kingdom. Injury, 36, pp.733-737.

[6] McLain, R., 2004. Functional outcomes after surgery for spinal fractures: Return to work and activity. 7 . Spine 29, 470-47, 29, pp.470-547.
[7] Road accident fatalities - statistics by type of vehicle - Statistics Explained, 2020.

[8] Bruns J, Jr, Hauser WA. The epidemiology of traumatic brain injury: A review. Epilepsia. 2003 ;44 :2-10. [PubMed] [Google Scholar]

[9] Thurman DJ, Alverson C, Dunn KA, et al. Traumatic brain injury in the United States: $A$ public health perspective. J Head Trauma Rehabil. 1999; 14:602-15. [PubMed] [Google Scholar]

[10]Ruchholtz, S., Ulrike, L., Debus, F., Mand, C., Siebert, H. and Kuhne, C., 2014. TraumaNetzwerk DGU1: Optimizing patient flow and management. Injury, Int. J. Care Injured, 45, pp.89-92.

[11]Lefering, R., Paffrath, T., Bouamra, O., Coats, T., Woodford, M., Jenks, T., Wafaisade, A., Nienaber, U. and Lecky, F., 2012. Epidemiology of in-hospital trauma deaths. 2012 Feb;38(1):3-9. Eur J Trauma Emerg Surg, 38(1), pp.3-9.

[12] Sturm, J., Pape, H. and Dienstknecht, T., 2013. Trauma care in Germany: an inclusive system. Clin Orthop Relat Res, 471(9), pp.2912-2923.

[13]Patton, G., Coffey, C., Sawyer, S., Viner, R., Haller, D., Bose, K. and et al, 2009. Global patterns of mortality in young people: a systematic analysis of population health data. The Lancet, 374(9683), pp.881-892.

[14] Mergoni, M, Saccani A, Salvadori A, Grattagliando $\mathrm{C}$, Paglari S, Bertone L, Peumonia in severe head injury. A prospective study. Minerva - Anesthesial, 1993, apr., 59/4, p. 187-192.

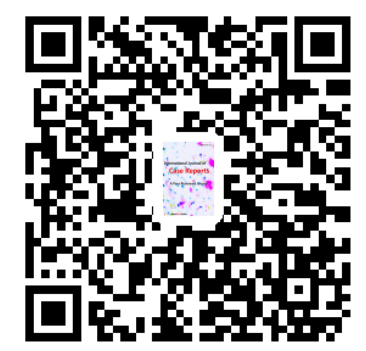

